\title{
Acoustic Considerations Regarding Pulsations during Large-scale Fire Tests in a Tunnel
}

\author{
ANDERS LÖNNERMARK and HAUKUR INGASON \\ Fire Technology \\ SP Swedish National Testing and Research Institute \\ Box 857 \\ SE-501 15 Borås, Sweden
}

\begin{abstract}
Large-scale fire tests have been carried out with Heavy Goods Vehicle (HGV) cargos in the Runehamar tunnel in Norway. During two of the fire tests, large pulsations of the gas flow inside the tunnel occurred. These pulsations were registered only when the measured heat release rates (HRR) were higher than $125 \mathrm{MW}$ - $135 \mathrm{MW}$. Two different periods of the pulsations were registered, short periods of about $4 \mathrm{~s}$ and longer periods of approximately $18 \mathrm{~s}$. In the article, the pulsations are presented and some plausible explanations are given, using an acoustic approach. There are several factors that can affect the pulsations, e.g., the size of the experimental set-up, the size of the fire, the thermal expansion at the site of the fire, the slope of the tunnel, the wind outside the tunnel, etc., but the calculations show that the oscillation periods are properties of the system, and can be what is referred to as thermoacoustic instabilities.
\end{abstract}

KEYWORDS: tunnel, fire, experiments, oscillations, pulsations, acoustics

\section{NOMENCLATURE}

\begin{tabular}{|c|c|c|c|}
\hline$A$ & Amplitude & $\operatorname{Re}$ & Reynolds number \\
\hline$a$ & Correlation parameter & St & Strouhal number \\
\hline$a$ & Speed of sound $(\mathrm{m} / \mathrm{s})$ & $T$ & Period (s) \\
\hline$b$ & Correlation parameter & $t$ & Time (s) \\
\hline$c_{\mathrm{p}}$ & Heat capacity const. p (J/kmol/K) & $U$ & Fuel velocity (m/s) \\
\hline$c_{V}$ & Heat capacity const. V $(\mathrm{J} / \mathrm{kmol} / \mathrm{K})$ & $u$ & Air velocity $(\mathrm{m} / \mathrm{s})$ \\
\hline$D$ & Diameter (m) & \multicolumn{2}{|l|}{ Greek } \\
\hline$f$ & Frequency (1/s) & $\gamma$ & $c_{p} / c_{V}$ \\
\hline Fr & Froude number & $\theta$ & Gas temperature $(\mathrm{K})$ \\
\hline$g$ & Gravitational acceleration $\left(\mathrm{m} / \mathrm{s}^{2}\right)$ & $\rho$ & Density $\left(\mathrm{kg} / \mathrm{m}^{3}\right)$ \\
\hline$k$ & Correlation coefficient & $\omega$ & Angular frequency (1/s) \\
\hline$L$ & Length (m) & \multicolumn{2}{|c|}{ subscripts } \\
\hline$M$ & Molecular mass (kg/kmol) & 0 & Ambient conditions \\
\hline$n$ & Mode & $b$ & Beat \\
\hline$p$ & Pressure $(\mathrm{Pa})$ & $m$ & Modulation \\
\hline & Gas constant $(\mathrm{J} / \mathrm{kmol} / \mathrm{K})$ & & \\
\hline
\end{tabular}

\section{INTRODUCTION}

In 2003, large-scale fire tests were performed with heavy goods vehicle (HGV) cargos in the Runehamar tunnel in Norway [1,2]. The tunnel is a two-way asphalted road tunnel that was taken out of use in the late 1980s. It is $1600 \mathrm{~m}$ long, $6 \mathrm{~m}$ high and $9 \mathrm{~m}$ wide, with a slope varying between $1 \%$ and $3 \%$. The main aim of the test series was to 
simulate a fire in a HGV trailer with ordinary goods and to study the fire development and peak HRR with such a cargo.

In two of these tests, periodical pulsations were observed during the most intense part of the fire. To the best of our knowledge, this type of pulsations has never previously been observed in large-scale testing or accidents in tunnels. Pulsations are a well-known phenomenon in enclosure fires, but not in tunnels. It is, therefore, of interest to explore and understand this phenomenon and put it into the perspective of tunnel safety. The pulsations of the air mass in the tunnel are described and theoretical analyses are carried out. The aim of this paper is to describe these pulsations and give some plausible explanation and theoretical background for them. Full details of the experimental series, the goods studied, and the conclusions of the project are provided elsewhere [1,2]. Only data of specific relevance to the discussion of the pulsation phenomena will be presented here.

\section{EXPERIMENTAL SET-UP}

In total, four tests were performed with a fire in an HGV set-up. In three tests, mixtures of different cellulosic and plastic materials were used, and in one test a commodity consisting of a mixture of furniture and fixtures was used. Table 1 summarizes the commodities tested and maximum heat release experienced in the four experiments. The pulsation phenomenon was only witnessed in Tests $\mathrm{T} 1$ and $\mathrm{T} 2$ and therefore more information will only be provided for these tests.

The centre of the fire was located $563 \mathrm{~m}$ from west entrance and the air flow direction in the tunnel was from east to west (see Fig. 1). In order to create a longitudinal flow inside the tunnel, two mobile fans were used. The fans were of the type Mobile Ventilation Unit - MVU 125/100 from B I G Innovative in Germany. These have an inner diameter of $1.25 \mathrm{~m}$ and six impeller blades. At $2000 \mathrm{rpm}$ these fans gave approximately $170000 \mathrm{~m}^{3} / \mathrm{h}$ and an axial thrust of $2600 \mathrm{~N}$. One fan was positioned approximately $10 \mathrm{~m}$ outside the eastern tunnel opening and one fan was positioned approximately $60 \mathrm{~m}$ into the tunnel. The two fans were able to create a longitudinal flow of $3 \mathrm{~m} / \mathrm{s}$ in the tunnel prior to ignition. The air speed was chosen to minimize the risk for back-layering.

Table 1. Description of the commodities and maximum HRR for each test.

\begin{tabular}{|c|l|c|c|}
\hline $\begin{array}{c}\text { Test } \\
\text { id }\end{array}$ & \multicolumn{1}{|c|}{ Description $^{\text {a }}$} & Total mass [kg] & $\begin{array}{c}\text { HRR }_{\text {max }} \\
\text { [MW] }\end{array}$ \\
\hline T1 & Wood pallets and plastic (PE) pallets & 11010 & 202 \\
\hline T2 & Wood pallets and mattresses (PUR) & 6930 & 157 \\
\hline T3 & Furniture and fixtures + rubber tires & $7750+800$ & 119 \\
\hline T4 & $\begin{array}{l}\text { Plastic cups (PS) in cardboard boxes on } \\
\text { wood pallets }\end{array}$ & 2850 & 66 \\
\hline
\end{tabular}

${ }^{\mathrm{a}} \mathrm{PE}=$ Polyethene, PUR = Polyurethane, PS = Polystyrene 


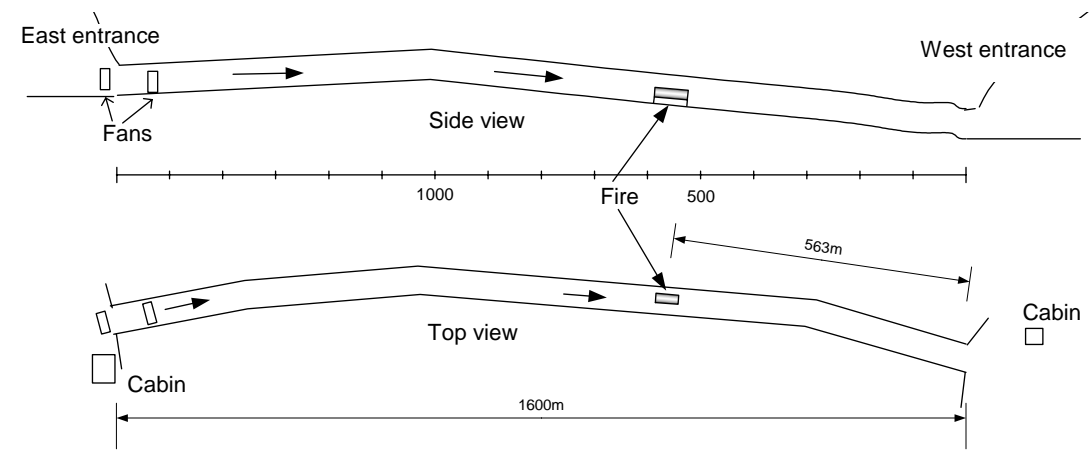

Fig. 1. Side and top view of the Runehamar tunnel and the position of the fire.

Ignition took place on the upstream side of the HGV-trailer (front). Two small ignition sources, consisting of fibreboard cubes soaked with heptane, were placed within the lowest wood pallets (adjacent to the flue between two pallets). The ambient conditions during the tests are presented in Table 2. The outside temperature varied somewhat between the tests, while the temperature inside the tunnel was essentially constant throughout the test series. The weather varied and it was rather windy during the first three tests.

Table 2. Temperature inside the tunnel and ambient conditions outside the tunnel.

\begin{tabular}{|l|c|c|c|c|}
\hline & Test T1 & Test T2 & Test T3 & Test T4 \\
\hline Temperature inside the tunnel $\left[{ }^{\circ} \mathrm{C}\right]$ & 10 & 11 & 10 & 11 \\
\hline Temperature $\left[{ }^{\circ} \mathrm{C}\right]$ & 14 & 9 & 9 & 11 \\
\hline Weather & Sun & Rain & Light rain & Cloudy \\
\hline Wind $^{\mathrm{a}}[\mathrm{m} / \mathrm{s}]$ & $\mathrm{NA}^{\mathrm{b}}$ & 4 & 4 & 0.2 \\
\hline Humidity $[\%]$ & NA & NA & 82.5 & 71 \\
\hline
\end{tabular}

NA = not available

${ }^{a}$ The wind was measured at an open space outside the tunnel near the eastern tunnel entrance. The dominant wind direction was from the west to the east.

${ }^{\mathrm{b}}$ The wind speed was not measured during this test, but the wind speed was estimated to be $3-5 \mathrm{~m} / \mathrm{s}$, i.e., comparable with the situation in T2 and T3.

\section{RUNEHAMAR TUNNEL MEASUREMENTS}

The heat release rate (HRR) was estimated using oxygen consumption calorimetry [3,4]. A number of different instruments were used to determine the HRR: five bi-directional pressure difference probes (McCaffrey and Heskestad [5]) each connected to a Furness mod FC0332 instrument; twelve thermocouples of type K with $0.25 \mathrm{~mm}$ diameter; two oxygen $\left(\mathrm{O}_{2}\right)$ analyzers of type PMA 10 and one of type Siemens Oxymat (lowest one); and two carbon dioxide $\left(\mathrm{CO}_{2}\right)$ /carbon monoxide (CO) analyzers of type BINOS. The gas velocity was also measured $50 \mathrm{~m}$ upstream of the fire, $3 \mathrm{~m}$ above the road surface. For this a bi-directional probe was used together with a pressure transducer (Furness FCO52 (ELITE)). In all cases the gas velocity was determined using the measured pressure difference $\Delta p$ for each probe and the corresponding gas temperature. The diameter of the probes used was $16 \mathrm{~mm}$ and the probe length was $32 \mathrm{~mm}$. The gas velocity was obtained from Eq. 1: 
where $k(\mathrm{Re})$ is a correction coefficient given by McCaffrey and Heskestad [5] which depends on the Reynolds number (Re). From the calibration curve presented by McCaffrey and Heskestad it is observed that $k(\operatorname{Re})$ is constant at 1.08 for Reynolds number greater than 2000. In the large-scale tests presented here, the Reynolds number was found to be in the range of $2000-3200$, which means that the $k$ could be set to 1.08 . The ambient values used in Eq. 1 were $\theta_{0}=283 \mathrm{~K}\left(10^{\circ} \mathrm{C}\right)$ and $\rho_{0}=1.24 \mathrm{~kg} / \mathrm{m}^{3}$. During test $\mathrm{T} 1$, the pressures at the downstream measurement station were, due to technical problems, sampled every 5 seconds instead of every second, as for the rest of the measurements. Therefore, the pressure data from this position in that test has not been used in the analyses. Temperatures were measured at several positions along the tunnel, from $100 \mathrm{~m}$ upstream of the fire (' $-100 \mathrm{~m}$ ') to a measurement station $458 \mathrm{~m}$ downstream of the fire, i.e., approximately $100 \mathrm{~m}$ from the west entrance. Details on the measurements, including results, of HRR, temperatures, gas analyses, and radiation are described elsewhere $[1,2,6,7]$. The results of interest for the analyses in this paper, including the measurements of the gas velocity and the resulting pulsations, are presented below.

\section{RESULTS}

In the first two fire tests, $\mathrm{T} 1$ and $\mathrm{T} 2$, a pulsation inside the tunnel was experienced during a time period when the HRR was higher than approximately 125-135 MW, which was reached only in the tests T1 and T2 (see Fig. 2a). This created a pulsating flow situation at the measuring station, where the measurements showed that the maximum velocity was pulsating in the range of $3 \mathrm{~m} / \mathrm{s}$ to $4.2 \mathrm{~m} / \mathrm{s}$ down to a minimum in the range of $1.5 \mathrm{~m} / \mathrm{s}$ to $3 \mathrm{~m} / \mathrm{s}$ (test T1 and T2). In Fig. 3a and 3b, plots of the velocity measured at the ceiling ( $5.1 \mathrm{~m}$ from the road surface) of the measuring station $+458 \mathrm{~m}$ are given for test T2. These velocity pulsations were measured at all heights at the measuring station and they were found to be highly periodical. The pulsations were only registered in the velocity (pressure) measurements. These pulsations also affected the calculated HRR since the mass flow rate, which is an important part in the HRR calculations, is calculated from the gas velocity [1]. The $\mathrm{O}_{2}$-measurements did not show any kind of pulsations (see Fig. 2b). The situation was the same also for other gas analyses, i.e., no pulsations although some small disturbances can be observed. The fast changes (short periods) might have been smoothed out in the tunnel or not registered due to the response time of the analysis system, but the pulsations with the longer periods cannot be seen either. This indicates that this is a pressure problem and not a combustion related problem even if the analyser response can not be taken as a very strong evidence. There is also the possibility that the heat release rate is oscillating, but to a much smaller degree than what is the case for the velocity. This would correlate with the case of a pulse burner, which is discussed in the next section, even if it is not only a question of heat release rate but also a question of heat transfer to the flow. 


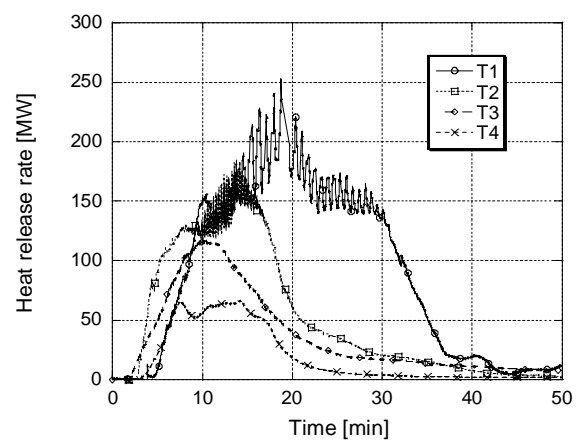

(a)

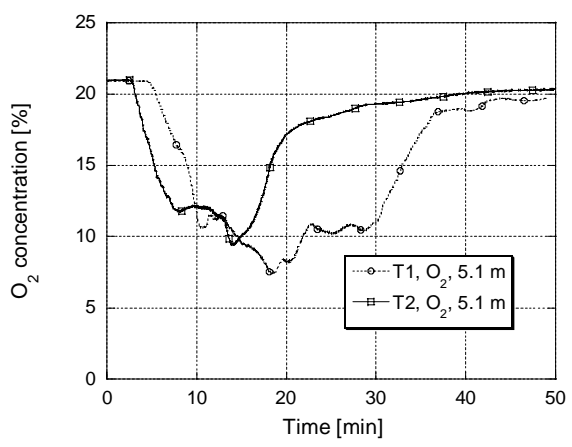

(b)

Fig. 2. (a) Heat release rates during the four tests. (b) $\mathrm{O}_{2}$ concentration at the downstream measurement station (5.1 $\mathrm{m}$ above the road surface) for the tests $\mathrm{T} 1$ and $\mathrm{T} 2$. Note that the HRR was calculated using the oscillating pressure signal to illustrate during what period the oscillations could be observed; the HRR values in the graph during this pulsation period are not the real ones.

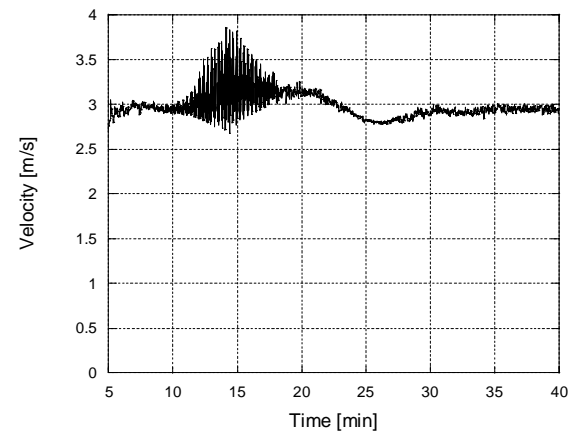

(a)

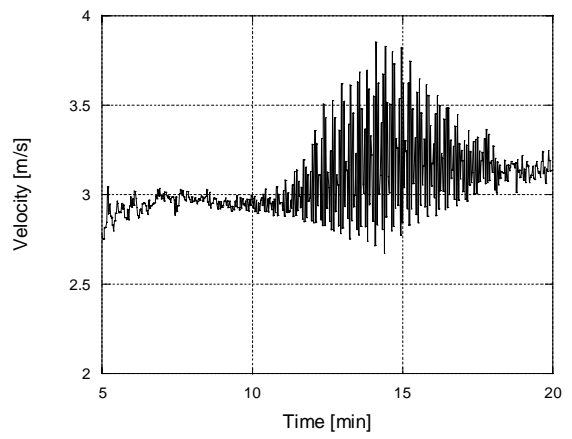

(b)

Fig. 3. Pulsating velocity during T2. Both figures show the velocity measurement $458 \mathrm{~m}$ downstream of the fire (5.1 $\mathrm{m}$ above the road), but with different scale.

The periods of the different pulsations during the fire tests are summarized in Table 3 . For the upstream measurements during T1 and both upstream and downstream measurements during T2 one can notice a short oscillation period between 4 and 5 seconds (median very close to $4 \mathrm{~s}$ ) and a longer period around $18 \mathrm{~s}$. When studying the shorter periods one notices that there are usually three or four $4 \mathrm{~s}$ periods followed by either one $6 \mathrm{~s}$ period or two $5 \mathrm{~s}$ period before another series of $4 \mathrm{~s}$ periods. 
Table 3. Periods of the oscillations registered during T1 and T2.

\begin{tabular}{|l|c|c|}
\hline \multicolumn{1}{|c|}{ Oscillation $^{\mathbf{a}}$} & $\begin{array}{c}\text { Average period } \\
\text { [s] }\end{array}$ & $\begin{array}{c}\text { Median period } \\
\text { [s] }\end{array}$ \\
\hline T1 upstream short & 4.84 & 4.02 \\
\hline T1 upstream long & 18.33 & 18.06 \\
\hline T1 downstream & - & - \\
\hline T2 upstream short & 4.47 & 4.08 \\
\hline T2 upstream long & 17.72 & 17.64 \\
\hline T2 downstream short & 4.49 & 4.08 \\
\hline T2 downstream long & 17.57 & 17.4 \\
\hline
\end{tabular}

${ }^{a}$ Observe that the period of the long oscillations is measure from one maximum to the next and is actually half the modulation period.

$\mathrm{b}$ The sampling period was longer in this case and is therefore left out of the analyses.

\section{AN ACOUSTIC EXPLANATION}

The measured periods of the pulsations were too long (too low frequencies) to be audible. Still an acoustic approach has been used to characterize the problem and to explain the properties of the pulsations and is presented in this section.

Combustion instabilities or pulsating flames are no new phenomenon. Byron Higgins is the first to have noted the type of combustion instability that usually is called a "singing flame” [8]. The discovery was made in 1777, but it was first in 1802 that he described the discovery in a letter to Nicholson's Journal [9,10]. Suggested explanations include production and condensation of water vapour, repeated detonations, decrease in pressure in the tube due to the draft, and velocities close to the critical velocity for the transition between streamline flow and turbulent flow [11]. These latter explanation lead to the assumption that the maintenance of vibrations cannot occur without draft, and therefore cannot occur in a horizontal pipe [12]. It has however been shown that this is not the case [11].

The best known explanation of the observed oscillations was given by Lord Rayleigh in 1878: “...If heat is given to the air at the moment of greatest condensation, or taken from it at the greatest rarefaction, the vibration is encouraged. On the other hand if heat is given at the moment of greatest rarefaction, or abstracted at the moment of greatest condensation, the vibration is discouraged..." [13]. This is referred to as Rayleigh's criterion and is still taken as valid today even if it is extended in the sense that the regions of oscillation are considerably broader than those described by Rayleigh's criterion [12].

The vibrations in an air tube can be initiated by some kind of disturbance. In the laboratory a small puff of air may be enough. In the large-scale, wind might have this initiating effect. Often, there is no obvious external reason for the start of the oscillations. [11]. Vortex shedding can be a reason for the production of oscillation and periodic heat release rate. The vortex shedding frequency can either coincide (approximately) with the second natural mode of the system or be large enough to force the system to follow the vortex shedding frequency or interact with the acoustic system in such a way that the vortex shedding is forced to attain the acoustic frequency [14]. The vortex shedding phenomenon has been widely studied when it comes to pool fires. In the review of pulsation of flames and pool fire performed by Malalasekera et al. [15], the authors give a relation between the Strouhal number, St, and the Froude number, Fr, on the form 
with the best fit for many experiments (burner diameter from $0.0074 \mathrm{~m}$ to $2.2 \mathrm{~m}$ ) giving $a=0.52$ and $b=-0.505$, which means that

$$
f=0.52 \cdot \frac{U}{D}\left[\frac{g D}{U^{2}}\right]^{0.505}
$$

The influence of the fuel velocity, $U$, is very limited and is therefore neglected in the analyses. Then the formula becomes similar to the one used in several cases in the literature:

$f=a \cdot D^{b}$

where the best fit for the same experiments as above gives $a=1.68$ and $b=-0.5$. These values are similar to those given by McCaffrey ( $a=1.6$ and $b=-0.5$ ) for fires with diameters between $2 \mathrm{~m}$ and $50 \mathrm{~m}$ [15]. If these values are used together with the dimensions of the set-up in the Runehamar fire tests, the frequency becomes $1 \mathrm{~Hz}$ if the width $(2.6 \mathrm{~m})$ is used and $0.5 \mathrm{~Hz}$ if the length $(10.5 \mathrm{~m})$ of the HGV mock-up is used. This corresponds to a period between 1 and 2 seconds, i.e., somewhat shorter than the measured period, but as mentioned it can still affect the oscillations by interacting with the acoustic system.

One type of pulse combustor that can be of special interest when comparing with the pulsations in the tunnel is the Rijke type pulse combustor or the Rijke tube. Rijke found in 1859 that if a piece of gauze was placed in the lower half of a wide tube and heated either by a Bunsen flame or an electric current, a musical vibration started $[8,10]$. The heat released from the gauze generates a periodic pulsation by the flow up in the tube. Rijke found that the maximum effect was achieved when the gauze was positioned one fourth of the length of the tube from its lower end. It is, however, possible to maintain oscillations also at other positions depending on the size of the flame [11]. Small flames must be inserted further than larger flames, into an air tube before they oscillate. The position and the size of the flame affects the pitch of the flame, and the pitches rise when the flame is moved further up into the air tube $[11,12]$. It has also been found that the frequency of the fundamental and the first harmonic can exist on the same time. Small flames have, on these occasions, been observed between the larger flames [12].

It is assumed that the tunnel can be represented by a tube open at both ends. This implies that there will be an amplitude maximum (pressure minimum) at the openings. In Fig. 4 the first three oscillation modes are presented. In the shadowed regions added heat is favourable for instability of the different modes. This is referred to as thermoacoustic instabilities (see e.g., [16]). These regions are defined as regions where the heat transfer to the flow fluctuates in phase with the pressure perturbation (see Rayleigh's criterion above). The phase of the heat transfer rate is dependent on the flow rate in the tunnel. This relation is described by a complex transfer function. This function has not been derived for the tunnel system here due to the limited data available. The fire (represented 
by dashed line (F)) was situated within such a region for the first harmonic and close to (with flames reaching into) one such region for the second harmonic.

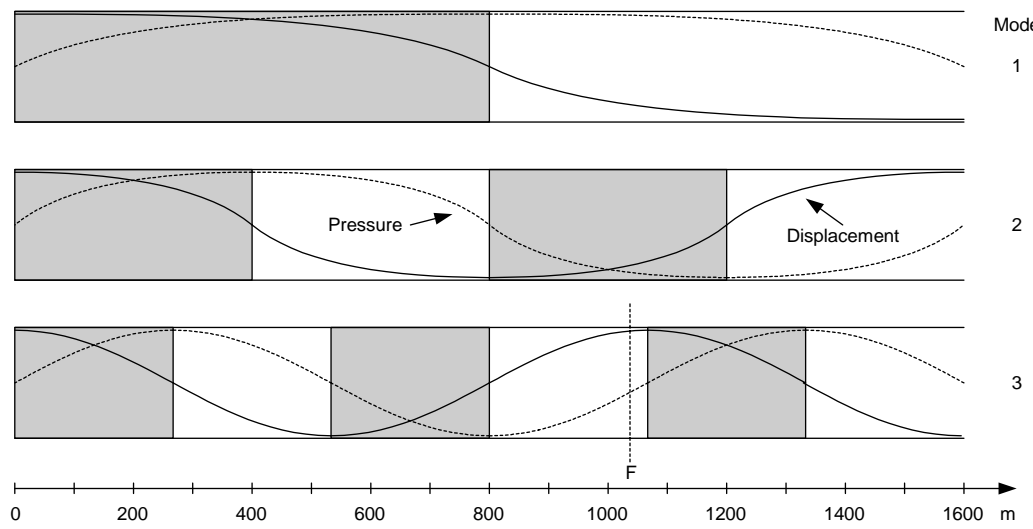

Fig. 4. The figures represent the amplitude for the first three modes of oscillations in a tube (the top one is the fundamental). The length scale of the tunnel is included for comparison. The vertical dashed line (F) represents the position of the centre of the fire.

The period of the oscillations can be described by

$T=\frac{2 L}{n a}, n=1,2,3, \ldots$

where $L$ is the length of the tube and $a$ is the velocity of sound. The time periods of the first modes of oscillation for a tube with the length of the Runehamar tunnel $(1600 \mathrm{~m})$ is given in Table 4. Note that the oscillation is dependent on the velocity of sound. This is in turn dependent on the temperature:

$a=\sqrt{\frac{\not R \theta}{M}}$ where

$\gamma=\frac{C_{P}}{C_{V}} \approx 1.4$ (varies with temperature),

$R$ is the gas constant, $\theta$ is the temperature in $\mathrm{K}$, and $M$ is the molar mass. This means that the time period is inversely proportional to the square root of the temperature, i.e., in the case of fire the time period would be shorter (the frequency higher). The effect of the temperature will be discussed later, but in the main acoustic calculations, a constant temperature has been used. When calculating the oscillations in a tube one often needs to consider also effects at the openings, so called end effects [14], but the end corrections are only a few fractions of a percent of the tunnel length for this long tunnel and have been neglected in these calculations. 
Table 4. Period for the first three modes of the oscillations in a $1600 \mathrm{~m}$ long tunnel (calculated for $283 \mathrm{~K}$ ).

\begin{tabular}{|l|c|}
\hline \multicolumn{1}{|c|}{ Mode (n) } & Period, $\boldsymbol{T}$ [s] \\
\hline 1 (Fundamental) & 9.49 \\
\hline 2 & 4.75 \\
\hline 3 & 3.16 \\
\hline
\end{tabular}

When combining two waves with the same amplitude and the angular frequencies $\omega_{1}$ and $\omega_{2}$, respectively, one can obtain a net wave expressed as

$p=A \cos (\bar{\omega} t) \cos \left(\omega_{m} t\right)$

where

$\bar{\omega}=\frac{1}{2}\left(\omega_{1}+\omega_{2}\right)$ and $\omega_{m}=\frac{1}{2}\left(\omega_{1}-\omega_{2}\right)$

is the average angular frequency and the modulation frequency, respectively. An example of such a wave is presented in Fig. 5, where it is compared with measurements. The graphs show a good correspondence between the behaviour of the signal in the measurements and the calculations, which means that the registered frequencies could be explained by the combination of two different waves. This is the case for all the oscillations (summarized in Table 3). The distance between each group maximum in the graph (Fig. 5) corresponds to twice the modulation frequency and this is correlated to what is called the beat frequency. The intensity of the wave oscillates with this frequency. Note that it is the period corresponding to the beat frequency $\left(\omega_{b}=2 \omega_{m}\right)$ that is reported in Table 3. Since it is periods that were discussed in the previous section it could be interesting to convert the average angular frequency and the beat frequency into corresponding periods. If the periods of the two original waves are $T_{1}$ and $T_{2}$, the resulting periods can be calculated as

$\bar{T}=\frac{2 T_{1} T_{2}}{T_{1}+T_{2}}$ and $T_{b}=\frac{T_{1} T_{2}}{T_{1}-T_{2}}$

Using the periods of the second and the third mode (4.75 s and $3.16 \mathrm{~s}$, respectively) would give $\bar{T}=3.8 \mathrm{~s}$ and $T_{b}=9.4 \mathrm{~s}$. The average period is close to the observed one, while the period of the beat is further away. However, a small change in the original waves can give a significant change in the resulting period due to the difference in the denominator. If the observed periods are assumed to be $\bar{T}=4 \mathrm{~s}$ and $T_{b}=18 \mathrm{~s}$, then one can calculate the periods of the original waves to be $4.5 \mathrm{~s}$ and $3.6 \mathrm{~s}$, respectively. 


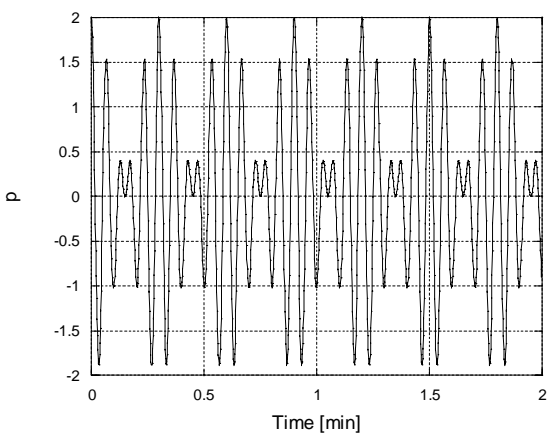

(a)

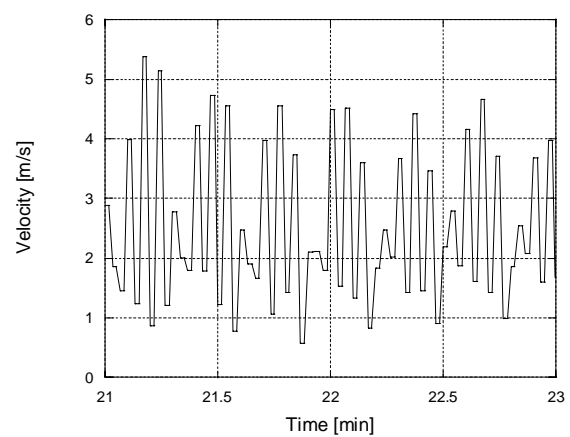

(b)

Fig. 5. Examples of beats (a) compared with two minutes of oscillations upstream of the fire in T1 (b). The short horizontal lines in the right hand graph are due to the data acquisition system and are not a physical phenomenon, even if non-linear effects are common in thermoacoustic oscillations.

The variation in the speed of sound due to the increased temperature has so far not been included. A heat transfer analysis gives an average temperature downstream of the fire that is approximately twice the ambient temperature (in K). Performing a length weighted calculation, with one temperature upstream and another downstream of the fire, gives a total speed of sound that is $15 \%$ higher than the speed of sound for a temperature of $283 \mathrm{~K}$. This corresponds to periods of $8.25 \mathrm{~s}, 4.13 \mathrm{~s}$, and $2.75 \mathrm{~s}$, respectively, for the first three oscillation modes described above, i.e., somewhat further away from the postulated values.

\section{DISCUSSION}

During the tests with the oscillations, it was rather windy and it was suggested that the fluctuations in the wind could have caused the oscillations inside the tunnel. Even though this might not be the fundamental explanation, the wind can affect the onset of the oscillations and constitute a force affecting the system.

The pulsations look somewhat similar to the pulsations of smoke through openings that sometimes can be seen in connection with vitiated fires in enclosures. This gave rise to the question if the fire in the tunnel could have been vitiated and thereby causing the pulsations. The fire was, however, not vitiated. Still, there can be situations with local lack of oxygen and this can be correlated to the pulsations.

There was a slight downhill slope where the fire was positioned (1\%) and the hot gases could cause a driving force against the flow in the tunnel. This will certainly be a force that has to be overcome by the fans and can probably, together with the pressure drop over the fire and the set-up, affect the oscillations; but this is unlikely to be the sole reason for the presence of the oscillations. In connection to this, one can also mention the very rapid increase in temperature of the gases, which leads to a rapid increase in the volume of the gases. This may also play an important role for the pressure drop of the fire and the set-up, but also for the vortex shedding. 
One interesting observation is the fact the oscillations start when the heat release rate increases above a certain value (between 125 and $135 \mathrm{MW}$ ). During the tests when higher HRR was not reached (tests T3 and T4), oscillations were not observed. This situation has not been modelled quantitatively, but the reason for this could be that when the fire is sufficiently large, the energy transferred to the acoustic mode is larger than the acoustic losses ("sound" radiation from the open tube ends and losses due to the acoustic boundary layer in the tunnel) and therefore is enough to maintain the pulsations. This can be related to the phenomena seen in connection with studies of the oscillating flame and the Rijke tube [16]. One interesting point is also that the amplitude of the oscillations is small in the beginning and largest when the heat release rate is the highest after which the amplitude decreases again.

As seen in Fig. 4, the fire was positioned within a region where heat addition to the flow favours instability of the second mode. The instability of the third mode can be explained by the flames extending into the corresponding region for the third mode. In the Rijke tube the position for a heater most often used is $1 / 4$ of the tube length from the upstream side and in these cases it is the first mode that is excited. The presented regions favouring instability, presented in the Fig. 4, can therefore explain why the oscillations can be related to the second and third modes.

\section{CONCLUSIONS}

Acoustic relations have been used to study the oscillation phenomena registered during the large-scale fire tests in the Runehamar tunnel. Two different types of periods were seen, one near $4 \mathrm{~s}$ and one around $18 \mathrm{~s}$. In this application, the acoustic approach seems to be able to explain the periods of the oscillations. The two observed periods can be the average period and the beating period, respectively, of two combined waves. The periods of these combined waves are close to the first two harmonics of the tunnel as an acoustic system, but they can also be affected by vortex shedding and by the position of the fire. The system needs, however, to be studied using a more detailed model to investigate what might affect the periods of the oscillations and the amplification and damping of these.

There is also a need for further investigation into the onset of the oscillations and the transfer function between the heat release and the flow system. Several factors probably collaborate to give rise to the oscillations as a combined effect. However, we feel that we have shown that a plausible explanation is the excitation of the natural frequencies of the system and that these oscillations can be referred to as thermoacoustic instabilities. It should be noted that the system here is not only defined by the tunnel itself but also by the distances between different objects (fire, fans, etc.) in the tunnel, the conditions at the tunnel opening, and the size of the fire. Future studies will focus on an investigation of the effect of impedance on the system as an alternative explanation for this phenomenon.

\section{ACKNOWLEDGEMENTS}

Dr Sven-Inge Möller is acknowledged for describing the use of pulse combustors today and Dr Bror Persson for interesting discussions regarding the pulsations. The work was funded by the Swedish Road Administration, the Swedish Rail Administration, the Swedish Rescue Services Agency, the Swedish Fire Research Board and the European Commission, Promat, Gerco, B I G, and the Norwegian Road Administration. 


\section{REFERENCES}

[1] Ingason, H., and Lönnermark, A., "Heat Release Rates from Heavy Goods Vehicles Trailers in Tunnels,” Fire Safety Journal, (accepted for publication).

[2] Lönnermark, A., and Ingason, H., "Gas Temperatures in Heavy Goods Vehicle Fires in Tunnels," Fire Safety Journal, (accepted for publication).

[3] Huggett, C., "Estimation of Rate of Heat Release by Means of Oxygen Consumption Measurements," Fire and Materials, 4, 2, pp. 61-65, 1980.

[4] Parker, W.J., "Calculations of the Heat Release Rate by Oxygen Consumption for Varios Applications,” National Bureau of Standards, NBSIR 81-2427, Gaithersburg, USA, 1982.

[5] McCaffrey, B.J., and Heskestad, G., "Brief Communications: A Robust Bidirectional Low-Velocity Probe for Flame and Fire Application," Combustion and Flame, 26, pp. 125-127, 1976.

[6] Lemaire, T., "Runehamar Tunnel Fire Tests: Radiation, Fire Spread and Back Layering,” International Symposium on Catastrophic Tunnel Fires (CTF), SP Report 2004:05, pp. 105-116, Borås, Sweden, 20-21 November, 2003.

[7] Brandt, A.B., "Presentation of Test Result from Large Scale Fire Tests at the Runehamar Tunnel," International Symposium on Catastrophic Tunnel Fires (CTF), SP Report 2004:05, pp. 117-120, Borås, Sweden, 20-21 November, 2003.

[8] Tyndall, J., Sound, Sixth ed., Longmans, Green, and Co., London, 1895.

[9] Higgins, B., "On the sound Produced by a Current of Hydrogen Gas Passing through a Tube,” Nicholson's Journal of Natural Philosophy, 50, pp. 129, 1802.

[10] Wood, A., Acoustics, Blackie \& Son, Glasgow, 1943.

[11] Jones, A.T., "Singing Flames," The Journal of the Acoustical Society of America, 16, 4, pp. 254-266, 1945.

[12] Richardson, E.G., "The Theory of the Singing Flame," Proc. Phys. Soc. (London), 35, pp. 47-55, 1922.

[13] Rayleigh, J.W.S., “The Explanation of Certain Acoustical Phenomena,” Nature, July, pp. 319-321, 1878.

[14] Putnam, A. A., Combustion-Driven Oscillations in Industry, American Elsevier Publishing Company Inc., New York, 1971.

[15] Malalasekera, W.M.G., Versteeg, H.K., and Gilchrist, K., "A Review of Research and an Experimental Study on the Pulsation of Buoyant Diffusion Flames and Pool Fires," Fire and Materials, 20, pp. 261-271, 1996.

[16] Matveev, K., "Thermoacoustic Instabilities in the Rijke Tube: Experiments and Modeling,” California Institute of Technology, Pasadena, California, USA, 2003. 